José Rafael MODOLO Hélio LANGONI ${ }^{1}$

Carlos Roberto PADOVANI ${ }^{2}$

Fábio Hiroto

SHIMABUKURO ${ }^{3}$

André de Oliveira

MENDONÇA ${ }^{3}$

Cassiano VICTORIA ${ }^{3}$

Welligton Borges da SILVA ${ }^{3}$

Correspondência para:

JOSÉ RAFAEL MODOLO

Departamento de Higiene Veterinária e

Saúde Pública

Faculdade de Medicina Veterinária e

Zootecnia

Universidade Estadual Paulista

Caixa Postal 524

18618-000 - Botucatu - SP

jrmodolo@fmvz.unesp.br

Recebido para publicação: 07/10/2004 Aprovado para publicação: 13/07/2005

\title{
Investigação soroepidemiológica de leptospirose canina na área territorial urbana de Botucatu, São Paulo, Brasil
}

\author{
1 - Departamento de Higiene Veterinária e Saúde Pública da Faculdade de \\ Medicina Veterinária e Zootecnia da Universidade Estadual Paulista, \\ Botucatu - SP \\ 2 - Departamento de Bioestatística do Instituto de Biociências da \\ Universidade Estadual Paulista, Botucatu - SP \\ 3 - Faculdade de Medicina Veterinária e Zootecnia da Universidade Estadual \\ Paulista, Botucatu - SP
}

\section{Resumo}

Palavras-chave: Cães.

Investigou-se, soroepidemiologicamente, a leptospirose em 775 cães do município de Botucatu - SP, em amostras de sangue, obtidas durante a campanha anual de vacinação anti-rábica, coletadas de 14 postos de vacinação, distribuídos geograficamente, representando o total de 45 postos de cinco regiões do Município, com uma área territorial urbana de $31 \mathrm{~km}^{2}$. Do total de amostras, 449 eram de machos, e 326, de fêmeas; 564 eram de animais sem raça definida, e 211, puros. As idades variavam de três meses a 20 anos. Para o diagnóstico, foi realizada a soroaglutinação microscópica, utilizando-se 12 sorovares de Leptospira spp. A comparação entre o resultado do exame sorológico e as variáveis epidemiológicas foi realizada pelos testes de Goodman e do Qui-quadrado, $\operatorname{com} \alpha=0,05$. Obtiveram-se 119 (15,3\%) amostras positivas, com reação para 11 sorovares, com maior importância para o canicola, em 48 (40,3\%) amostras, e pyrogenes, em 41 (34,5\%). Pela análise estatística, encontrou-se diferença significativa em relação à raça - positividade em $17,7 \%$ dos animais sem raça definida e em $9,0 \%$ dos demais - e sexo-positividade em 18,4\% de machos e $11,0 \%$ de fêmeas. Quanto à idade, não houve diferença significativa, com distribuição uniforme entre as faixas etárias estabelecidas.

\section{Introdução}

A leptospirose é uma doença zoonótica de ocorrência mundial, causada por diferentes sorovares de Leptospira spp. Hoje, é considerada uma infecção emergente $^{1,2}$. As leptospiras patogênicas foram formalmente classificadas como membros da espécie Leptospira interrogans, sendo, agora, agrupadas em sete espécies, compreendendo mais de 200 sorovares, conhecidos e identificados no mundo todo ${ }^{1}$.

O homem, os animais domésticos e inúmeros animais selvagens são afetados pela leptospirose, sendo referidas duas categorias da doença, com implicações clínicas diferentes: uma, quando o animal é infectado com um sorovar hospedeiro-adaptado, tornando-se reservatório, e a outra, quando animais susceptíveis são expostos a sorovares hospedeiros não adaptados, causando a doença acidental, forma comum aos humanos ${ }^{3}$. Nas duas situações, os animais infectados eliminam as leptospiras pela urina, por um período de semanas a meses, contaminando o ambiente ${ }^{4}$.

Entre os animais domésticos, o cão tem um papel importante na epidemiologia da leptospirose, principalmente em países tropicais ${ }^{4,5,6}$.

Os sorovares predominates na infecção canina parecem ser, ainda, o canicola, 
sorovar adaptado à espécie, e o icterohaemorrhagiae, que tem como principal reservatório o rato doméstico (Rattus norvegicus), infectando o cão acidentalmente ${ }^{1}$.

Em pesquisas sorológicas feitas na cidade de Salvador-BA, examinando-se amostras coletadas durante a campanha de vacinação anti-rábica, obtiveram-se 21,6\% (93/430) de casos positivos. Os autores verificaram maior importância para os sorovares icterohaemorrhagiae e canicola ${ }^{5}$.

Em trabalho realizado em cães de rua, em Porto Rico, verificaram-se aglutininas antileptospíricas em 62,9\% (73/116) dos animais, ocorrendo maior número de reações para o sorogrupo Icterohaemorrhagiae?

Estudos recentes revelam a importância de outros sorovares para os cães. Em pesquisa realizada na Austrália, considerando-se títulos iniciais de $50 \mathrm{UI}$, obteve-se positividade em 9,8\% (49/501) dos cães, com prevalência para os sorovares icterohaemorrbagiae e grippotyphosa. ${ }^{8}$. No Chile, verificaram-se taxas de 38,33\% (23/60) de positividade em cães atendidos no Hospital Veterinário da Universidade de Concepción, sendo mais freqüentes os sorovares canicola e pyrogenes.

Também se obteve, em outro estudo sorológico feito em Patos-PB, uma prevalência de aglutininas antileptospíricas em 20\% (23/114) de cães domiciliados, atendidos na Clínica de Pequenos Animais do Hospital Veterinário da Universidade Federal da Paraíba; observou-se maior freqüência para os sorovares autumnalis, grippotyphosa, butembo e australis ${ }^{10}$.

Levando-se em consideração a importância da etiologia nas infecções leptospíricas, objetivou-se conhecer a soroprevalência da leptospirose na população canina, em Botucatu, e verificar o perfil dos sorovares de Leptospira spp, frente ao sexo, idade e raça de cada animal.

\section{Materiais e Métodos}

O município de Botucatu localiza-se na região centro-sul do Estado de São Paulo
( $22^{\circ} 52^{\prime}$ de latitude sul e $48^{\circ} 26^{\prime}$ de longitude oeste); está cerca de 805 metros acima do nível do mar e possui uma área territorial de $1.483 \mathrm{Km}^{2}$. O clima é subtropical úmido, com invernos secos e verões quentes, e a precipitação pluviométrica é de $1.250 \mathrm{~mm}$. A vegetação consiste em mata pluvial e cerrado. A população é estimada em 108.306 habitantes, de acordo com o censo do Instituto Brasileiro de Geografia e Estatística $(\mathrm{IBGE})^{11}$.

Durante a campanha anual de vacinação anti-rábica, coordenada pela disciplina de Planejamento em Saúde Animal e Saúde Pública, em conjunto com a Prefeitura Municipal de Botucatu, coletaramse 775 amostras de sangue de cães. As amostras, aproximadamente $5,0 \%$ de um total de 15.574 cães vacinados durante a campanha, foram coletadas em 14 postos de vacinação, representativos do total de 45 postos, distribuídos nas cinco regiões do Município, com uma área territorial urbana de $31 \mathrm{~km}^{2}$ (Prefeitura Municipal de Botucatu, Secretaria de Turismo, comunicação pessoal, 2000). Para o procedimento de alocação do animal ( $2^{\circ}$ estágio), considerou-se o plano de amostragem sistemática, sob a definição de que, a partir do animal selecionado, os dois seguintes seriam descartados. Quanto aos postos de coleta, procedeu-se, em primeiro estágio, ao sorteio geográfico de 14 postos de vacinação. $\mathrm{O}$ tamanho amostral, 775 cães, foi determinado considerando-se a taxa de $10 \%$ de erro de estimação, com nível de $95 \%$ de confiança ${ }^{12}$.

O sangue obtido de cada animal, cerca de $10 \mathrm{~mL}$, pela punção venosa cefálica ou jugular, com seringa descartável de $10 \mathrm{~mL}$ e agulha $30 \times 8 \mathrm{~mm}$, foi transferido para um tubo de vidro de $15 \mathrm{~mL}$ e, posteriormente, enviado ao Laboratório de Diagnóstico de Zoonoses da FMVZ - UNESP - Botucatu, onde foi centrifugado a $3.000 \mathrm{RPM}$, por dez minutos, para obtenção do soro, que foi acondicionado em microtubo de plástico de $1,5 \mathrm{~mL}$ e mantido em freezer a $-20^{\circ} \mathrm{C}$, até o momento do exame. O exame foi realizado pelo método de soroaglutinação 
microscópica, utilizando-se 12 sorovares, representantes de dez sorogrupos de Leptospira spp., a saber: australis, bratislava, autumnalis, canicola, cynopteri, djasiman, grippotyphosa, copenhageni, icterohaemorrhagiae, pomona, pyrogenes e hardjo, mantidos no referido laboratório, em meio líquido "EMJH" com albumina bovina "Tween 80"13. Consideraram-se como reagentes títulos iguais ou superiores a $100 \mathrm{UI}^{1}$.

Os dados referentes a sexo, idade e raça de cada animal foram obtidos das fichas de arquivo, preenchidas no momento da coleta de sangue, com a entrevista do proprietário.

A análise das associações entre o resultado do exame sorológico e as variáveis epidemiológicas foi realizada pelo teste de Goodman, para contrastes entre e dentro de populações multinomiais ${ }^{14,15}$. Para o estudo da existência de distribuição preferencial sistematizada, segundo as categorias de respostas das variáveis sexo, raça e idade, utilizou-se o teste do Quiquadrado para uma amostra ${ }^{16}$.

\section{Resultados}

Do total de 775 (100\%) soros trabalhados, foram reagentes $119(15,3 \%)$ cães.

Dos sorovares testados, apenas um, cynopteri, do sorogrupo cynopteri, não reagiu para nenhuma das amostras. Observa-se, na tabela 1, a freqüência dos sorovares, sem discriminação de títulos, com co-infecção e sem reação cruzada.

Quando se considerou a discriminação de títulos, sem co-infecção e com reação cruzada, a freqüência dos sorovares encontra-se na tabela 2.

O maior título, e único, nas duas situações, foi 3200 UI para o sorovar pyrogenes. Estatisticamente, houve diferença significativa no número de animais reagentes: segundo o sexo, com $\chi^{2}=8,05(\mathrm{p}<0,05)$ (Tabela 3); e, segundo a raça, com $\chi^{2}=8,99$ $(\mathrm{p}<0,05)$ (tabela 4).

Com relação à idade, distribuída em quatro faixas etárias, não houve diferença significativa, com animais de três meses até

Tabela 1 - Distribuição de títulos de anticorpos antileptospira em cães, na área territorial urbana de Botucatu, São Paulo, Brasil ${ }^{a}$

\begin{tabular}{cccccccc}
\hline & \multicolumn{7}{c}{ Títulos de anticorpos (UI) } \\
\hline Sorovares & 100 & 200 & 400 & 800 & 1600 & 3200 & Total \\
australis & № & № & № & № & № & № & № $(\%)$ \\
bratislava & 2 & 2 & - & - & - & - & $4(1,8)$ \\
autumnalis & 10 & 2 & 1 & 2 & - & - & $12(5,5)$ \\
canicola & 29 & 22 & 19 & 3 & 4 & - & $14(6,4)$ \\
cynopteri & - & - & - & - & - & - & $77(35,4)$ \\
djasiman & 1 & - & - & - & - & - & 0 \\
grippothyposa & 1 & - & 1 & - & - & - & $1(0,5)$ \\
copenhageni & 9 & 5 & 3 & - & - & - & $17(0,9)$ \\
icterohaemorrhagiae & 5 & 11 & 4 & 2 & - & - & $22(10,1)$ \\
pomona & 3 & - & 1 & - & 1 & - & $5(2,3)$ \\
pyrogenes & 16 & 21 & 8 & 5 & 9 & 1 & $60(27,5)$ \\
hardjo & 3 & 1 & - & - & - & - & $4(1,8)$ \\
Total & 86 & 66 & 37 & 13 & 15 & 1 & $218(100)$
\end{tabular}

a Considerou-se que um mesmo animal pode estar infectado para mais de um sorovar de Leptospira spp. $\chi^{2}=348,02(p<0,05)$. 
Tabela 2 - Distribuição de títulos de anticorpos antileptospira em cães, na área territorial urbana de Botucatu, São Paulo, Brasil b

\begin{tabular}{cccccccc}
\hline & \multicolumn{7}{c}{ Títulos de anticorpos (UI) } \\
\hline Sorovares & 100 & 200 & 400 & 800 & 1600 & 3200 & Total \\
australis & № & № & № & № & № & № & № $(\%)$ \\
bratislava & 1 & 1 & - & - & - & - & $2(1,7)$ \\
autumnalis & 3 & - & - & 2 & - & - & $5(4,2)$ \\
canicola & 5 & 2 & - & - & 1 & - & $8(6,7)$ \\
cynopteri & 12 & 15 & 15 & 2 & 4 & - & $48(40,3)$ \\
djasiman & - & - & - & - & - & - & 0 \\
grippothyposa & - & - & - & - & - & - & 0 \\
copenhageni & - & - & - & - & - & - & 0 \\
icterohaemorrhagiae & 4 & 2 & - & - & - & - & $6(5,0)$ \\
pomona & 1 & 3 & 1 & - & - & - & $5(4,2)$ \\
pyrogenes & 1 & - & - & - & 1 & - & $2(1,7)$ \\
hardjo & 4 & 14 & 9 & 4 & 9 & 1 & $41(34,5)$ \\
Total & 1 & 1 & - & - & - & - & $2(1,7)$ \\
& 32 & 38 & 25 & 8 & 15 & 1 & $119(100)$ \\
\hline
\end{tabular}

b Para os animais que reagiram para mais de um sorovar, considerou-se como infectante o sorovar de maior título

Tabela 3 - Distribuição de cães reagentes à Leptospira spp., de acordo com o sexo, na área territorial urbana de Botucatu, São Paulo, Brasil

\begin{tabular}{|c|c|c|c|c|}
\hline Sexo & $\begin{array}{c}\text { Reagente } \\
\mathrm{N}^{\mathrm{o}}\end{array}$ & $\begin{array}{c}\text { Não reagente } \\
N^{\circ}{ }^{\circ}\end{array}$ & $\begin{array}{c}\text { Total } \\
\text { № }\end{array}$ & $\%$ Reagente \\
\hline Macho & 83 & 366 & 449 & 18,4 \\
\hline Fêmea & 36 & 290 & 326 & 11,0 \\
\hline Total & 119 & 656 & 775 & 15,3 \\
\hline
\end{tabular}

$\chi^{2}=8,05(p<0,05)$

Tabela 4 - Distribuição de cães reagentes à Leptospira spp., de acordo com a raça, na área territorial urbana de Botucatu, São Paulo, Brasil

\begin{tabular}{ccccc}
\hline Raça & Reagente & Não reagente & Total & \% Reagente \\
\hline Sem raça definida & № & № & 564 & 17,7 \\
Com raça & 100 & 464 & 211 & 9,0 \\
Total & 19 & 192 & 775 & 15,3 \\
\hline
\end{tabular}

$\chi^{2}=8,99(p<0,05)$

Tabela 5 - Distribuição de cães reagentes à Leptospira spp., de acordo com a idade, na área territorial urbana de Botucatu, São Paulo, Brasil

\begin{tabular}{|c|c|c|c|c|}
\hline Idade (anos) & $\begin{array}{c}\text { Reagente } \\
\quad №\end{array}$ & $\begin{array}{c}\text { Não reagente } \\
\mathrm{N}^{\circ} \mathrm{O}\end{array}$ & $\begin{array}{c}\text { Total } \\
\text { № }\end{array}$ & $\%$ Reagente \\
\hline $0,25-3,3$ & 66 & 379 & 445 & 14,8 \\
\hline $3,4-6,6$ & 35 & 157 & 192 & 18,2 \\
\hline $6,7-9,9$ & 11 & 75 & 86 & 12,8 \\
\hline$\geq 10,0$ & 4 & 32 & 36 & 11,1 \\
\hline Sem informação & 3 & 13 & 16 & 18,7 \\
\hline Total & 119 & 656 & 775 & 15,3 \\
\hline
\end{tabular}

$\chi^{2}=2,80(p>0,05)$ 
20 anos de idade reagindo igualmente, com $\chi^{2}=2,80(\mathrm{p}>0,05)($ tabela 5).

\section{Discussão}

A freqüência de aglutininas antileptospíricas $(15,3 \%)$ em cães, no município de Botucatu-SP, revela taxas médias, quando comparada à obtida em outras pesquisas. Considerando-se que, na maioria dos trabalhos, as condições epidemiológicas e o padrão de amostragem utilizado na seleção, presença ou ausência da doença no local de investigação e o "status" vacinal e social do animal são variáveis, tornase difícil a obtenção de conclusões. Em estudo semelhante, na cidade de Salvador$\mathrm{BA}$, obtiveram-se taxas maiores de reação $(21,6 \%)^{5}$. Em relação a outros países, observaram-se índices mais altos em Porto Rico, com uma porcentagem de $62,9 \%$ em cães de rua ${ }^{6}$. Freqüências menores foram verificadas na Austrália, com 9,8\% .

A distribuição dos sorovares, quando considerada a co-infecção, sem reação cruzada e com discriminação de títulos, mostra maior importância para os sorovares canicola e pyrogenes. Em estudo realizado no Chile, é relatada a mesma importância para esses sorovares, em animais atendidos no Hospital Veterinário da Universidade de Concepción?.

Os sorovares mais freqüentes também foram canicola e pyrogenes, quando se desconsiderou a co-infecção, em que a reação de mais de um sorovar para o mesmo animal foi avaliada como reação cruzada, escolhendo-se, como possível infectante, o sorovar de maior título ${ }^{1}$.

A interpretação de resultados sorológicos para leptospirose torna-se complicada por inúmeros fatores, tais como: reação cruzada de anticorpos, títulos de anticorpos induzidos pela vacinação e dificuldade do consenso sobre quais títulos de anticorpos são indicativos da infecção ativa. Em geral, cães vacinados desenvolvem, baixos títulos de anticorpos aglutinantes (100 a $400 \mathrm{UI})$, os quais persistem de um a três meses, após a vacinação ${ }^{1}$, dados que não poderiam ser extrapolados para este estudo, uma vez que, nesta pesquisa, não foi considerado o "status" vacinal dos animais amostrados.

O icterohaemorrhagiae, sorovar comum em ratos e responsável por surtos epidêmicos, principalmente em regiões tropicais com altos índices pluviométricos ${ }^{2}$, mostra-se menos importante, o que pode ser explicado pelo fato de que, no município de Botucatu, mesmo em épocas de chuvas fortes, não ocorrem alagamentos e enchentes, diminuindo a probabilidade de infecções por esse sorovar.

Outros sorovares, como copenhageni, autumnalis e bratislava, considerados emergentes ou reemergentes, em outros países, revelaram certa importância neste trabalho, bem como em outros estudos ${ }^{1,2,10}$.

A porcentagem dentre cães machos foi significativamente maior, corroborando os mesmos resultados verificados por outros autores $^{5,9}$, explicados pelo comportamento sexual do macho, lambendo e cheirando a genitália da fêmea, e pelo hábito de marcação territorial, cheirando a urina de outros animais.

Quanto à raça, a mesma diferença foi verificada, com o dobro de reagentes dentre os animais sem raça definida, em relação aos com raça; constatou-se o mesmo em outro estudo realizado na cidade de Salvador-BA ${ }^{5}$. A justificativa para essa diferença é que, aqueles animais têm acesso livre à rua, enquanto que os com raça vivem domiciliados, na maioria das vezes.

Quanto à idade, os animais de três meses até 20 anos mostraram-se igualmente reagentes, sem diferença significativa. Esses dados são diferentes dos verificados por outros autores, que mostram maior freqüência em animais de maior idade ${ }^{9}$, o que pode ser explicado pelas maiores chances de contato com fontes de infecções.

Apesar de a presença de aglutininas antileptospíricas em cães domésticos não se apresentar alta (15,3\%), no município de Botucatu-SP, com títulos baixos em sua 
maioria, indicando, em muitos casos, somente infecção ou estágio agudo da doença ${ }^{10}$, há de se considerar que muitos animais permanecem portadores por um determinado tempo, eliminando leptospiras pela urina, no ambiente, o que possibilita que outros animais e o próprio homem sejam infectados. É importante ressaltar que os cães estão se contaminando, contribuindo para a disseminação das leptospiras e podendo colocar em risco a saúde da população.

\title{
Seroepidemiological inquiry of canine leptospirosis in the urban area from Botucatu, Sao Paulo State, Brazil
}

\begin{abstract}
Leptospirosis was seroepidemiologically investigated in $775 \mathrm{dogs}$ from Botucatu, state of Sao Paulo, in blood samples collected during the annual anti-rabies vaccination campaign. The samples were collected from 14 out of the 45 vaccination stations geographically distributed in five different municipal districts, with a territorial urban area of $31 \mathrm{Km}^{2}$. Samples were taken from 449 males and 326 females; 564 were of undetermined breed, and 211 were pedigree animals; ages ranged from 3 months to 20 years. Diagnosis included the microscopic serum agglutination tests using 12 Leptospira spp serovars. Serological results and epidemiological variables were compared using Goodman and chi-square tests, with $\chi=0.05$. There were $119(15.3 \%)$ positive samples for 11 serovars; the most important was canicola with 48 (40.3\%), followed by pyrogenes 41 (34.5\%). Statistical analysis showed significant difference for: breed, $17.7 \%$ positive in undetermined breeds and $9.0 \%$ in pedigrees; and sex, $18.4 \%$ positive in males and $11.0 \%$ in females. There was no statistically significant difference in relation to age with uniform distribution of positives.
\end{abstract}

\section{Referências}

1 BOLIN, C. A. Diagnosis of leptospirosis: a reemerging disease of companion animals. Semin. Vet. Med. Surg. (Small Animal), v. 11, n. 3, p. 166-171, 1996.

2 LEVETT, P. N. Leptospirosis: re-emerging or rediscovered disease?. J. Med. Microbio., v. 48, n. 2, p. 417-418, 1999.

3 HEATH, S. E.; JOHNSON, R. Leptospirosis. J. Am. Vet. Med. Assoc., v. 205, n. 11, p. 1518-1523, 1994.

HANSON, L. E. Leptospirosis in domestic animals: the public health perspective. J. Am. Vet. Med. Assoc., v. 181, n. 12, p. 1505-1509, 1982.

4 CALDAS, E. M.; DORIA, J. D.; MARTINS, M. A. Immunological inquiry for the epidemiology of leptospirosis in Canis familiares in Salvador, Bahia, Brazil. Int. J. Zoon., v. 4, n. 2, p. 103-110, 1977.

5 MONTES, A. S.; DIMAS, J. S.; RODRIGUEZ, F. J. P. La rata y el perro, importantes vectores de la leptospirosis en explotaciones pecuarias de $\mathrm{Cd}$. Guzmán, Jalisco. Rev. Cub. Med. Trop., v. 54, n. 1, p. 21-23, 2002.

6 FARRINGTON, N. P.; SULZER, K. R. Canine
Key-words: Dogs. Leptospiras. Urban area. leptospirosis in Puerto Rico. Int. J. Zoon., v. 9, n. 1, p. 45-50, 1982.

7 DICKESON, D.; LOVE, D. N. A serological survey of dogs, cats and horses in South-eastern Australia for leptospiral antibodies. Aust. Vet. J., v. 70, n. 10, p. 389-390, 1993.

8 PINEDA, M.; LÓPEZ, J.; GARCÍA, M. Frecuencia de leptospirosis en perros al test de aglutinación microscópica en Chillán-Chile. Arch. Med. Vet., v. 28, n. 1, p. 59-66, 1996.

9 ALVES, C. J. et al. Avaliação dos níveis de aglutininas anti-leptospira em cães no município de Patos-PB, Brasil. Rev. Bras. Ci. Vet., v. 7, n. 1, p. 17-21, 2000.

10 INSTITUTO BRASILEIRO DE GEOGRAFIA E ESTATÍSTICA. Base de Informações Municipais: Malha Municipal Digital 2000. Disponível em: < $\underline{\text { http:// }}$ www.ibge.gov.br >. Acesso em: 20 jun. 2004.

11 COCHRAN, W. Sampling Techniques. 3. ed. New York: John Wiley; 1977.

12 BRASIL. Ministério da Saúde. Fundação Nacional de Saúde. Centro Nacional de Epidemiologia. Coordenação de Controle de Zoonoses e Animais Peçonhentos. Manual de leptospirose, 2. ed. Brasília: Fundação Nacional de Saúde, 1995. 98 p. 
13 GOODMAN, L. A. Simultaneous confidence intervals for contrasts among multinomial populations. Ann. Math. Stat., v. 35, p. 716-725, 1964.

14 GOODMAN, L. A. On simultaneous confidence intervals for multinomial proporcions. Technometrics., v. 7, p. 247-254, 1965.

15 STREINER, D. L.; NORMAN, G. R. Biostatistics: the base essentials. St. Louis: Mosby - Year - Book, 1994. 260 p. 